\title{
The process of clinical consultation is crucial to patient outcomes and safety: 10 quality indicators s $^{* \prime \prime}$
}

\author{
Author: Gordon Caldwell ${ }^{\mathrm{A}}$
}

In this paper, I discuss the central importance of the clinical consultation to defining the potential outcomes for an episode of care. The consultation is also crucial to patient safety. Yet the processes of clinical consultations on ward rounds and outpatients have attracted little attention in terms of ergonomics or research. I propose 10 quality indicators that would be simple to measure and could be used to improve clinical consultations. Better consultations would reduce the possibility of misdiagnosis and also improve patient outcomes, patient experience, patient safety and staff satisfaction. It is high time we improved clinical consultations in hospital settings.

KEYWORDS: Clinical consultation, misdiagnosis, clinical reasoning, patient outcomes, patient experience

\section{Background}

During a new patient consultation the clinician is firstly trying to work out whether the patient is ill. Then the clinician is trying to answer 'What is the diagnosis?' so that the appropriate investigations, treatment and review can be planned. Expert clinicians know that the majority of the information directing towards the correct diagnosis comes from attentive listening to the patient's history of the presenting complaint, set in the context of the patient's life and known medical conditions. Physical examination adds some further useful information and tests are used to confirm or refute possibilities. As the clinician listens to and examines the patient, their brain is full of clinical reasoning, which is high-level thinking around possible diagnoses. Their clinical reasoning directs the closed questions and focused physical examination. The clinician is doing two high-level mental activities simultaneously: attentive listening and clinical reasoning. Most people cannot even do attentive listening to someone else, let alone high-level thinking at the same time!

It follows that if the clinician elicits a poor history or physical examination, they may well arrive at the incorrect diagnosis. Similarly, if their mind is not free for clinical reasoning again, they may well arrive at the incorrect diagnosis. If the diagnosis is

Author: ${ }^{\text {A }}$ consultant, Lorn and Islands District General Hospital, Oban, UK incorrect, the treatment will be incorrect and recovery delayed or derailed completely.

\section{Outcome or process measurements?}

There is a lot of debate in healthcare about whether we should measure process or outcome. I argue that the process of clinical consultation defines the possible outcomes. We cannot know the possible outcomes until we know the diagnosis. The clinical consultation is when the diagnosis is made correctly or incorrectly. If an incorrect diagnosis is made, a good outcome can only arise by good luck eg if the clinician believes that the diagnosis is a urine infection but is actually pneumonia, by good luck the antibiotic might treat the pneumonia. However, an incorrect diagnosis usually results in delayed and prolonged care, iatrogenic harm or even an avoidable death.

A review consultation is as important. The clinician has decided on a likely or definite diagnosis and recommended a treatment plan. The clinician will have an expected trajectory of progress in their mind. They have to determine whether the patient has followed that trajectory or fallen away. Even if the patient is making good progress the clinician should still re-question the diagnosis. If the patient has fallen away from the expected trajectory then the diagnosis must be reviewed as well as determining whether the treatment plan has been executed. Revising a diagnosis is a major psychological effort and again the clinician's mind must be free for high-level clinical reasoning.

\section{The study of clinical consultations}

In modern healthcare, the clinical consultation is almost completely overlooked and ignored. We are under pressure to see more and more patients, to record more and more notes, to complete more and more pro formas, to meet relentless performance targets, follow guidelines and fill in the most recent form that is meant to avoid some recent harms in the systems of care or provide statistics to management. At the same time, we have to battle either with illegible paper notes (charts) or hugely inefficient software purporting to be electronic patient records. These battles can use up all of our brain's cognitive capacity, so that there is no brain power left for clinical reasoning.

High-level thinking usually requires a quiet controlled environment like a library or conference room; interruptions must be kept to a minimum, the brain must be refreshed by regular refreshment breaks and important information must be to hand. Again, there is so much emphasis on evidence-based treatment, 
Box 1. The 10 quality indicators for clinical

consultation. Reproduced with permission from Launer

J. Is there a crisis in clinical consultations? Postgrad Med J 2017:93:58.

> The patient should be as prepared as possible.

> The clinician should be as prepared as possible.

$>$ The clinician should know the person before making the person into a patient.

> The consultation should feel unhurried for the patient and clinician.

> The clinician should be able to give undivided attention to the patient.

> The clinician should be able to hear themself think.

> There should be ready supply of information into the consultation.

$>$ Confidentiality and dignity should be maintained.

> The clinician should be regularly refreshed.

> The patient should be encouraged to have an important othe person participate in the consultation.

but so little emphasis on the process to correct diagnosis. We need a lot of information readily available to make the correct diagnosis in the patient's context. Simple facts like known pre-existing diagnoses, medications and results of tests can be impossible to find or, once found, difficult to read and assimilate. Clinical consultations rarely feel like well-prepared business-like meetings. If we cannot ensure that clinicians' brains are free for high-level clinical reasoning, we should not be surprised if patient outcomes and experiences are poor, bad or even fatal.

\section{Ten quality indicators for clinical consultations}

In 2017, I proposed 10 quality indicators for clinical consultation (Box 1). ${ }^{1}$ Some of these are not easy to measure, but we must learn to measure what we value not just value what is easy to measure. My belief is that if healthcare providers placed as much emphasis and resource on my 10 quality indicators for clinical consultation as they have put on venous thromboembolism (VTE) prophylaxis or the 4-hour emergency department (ED) target, we would have substantial improvements in patient outcomes and experience within 2 years. Costs would also come down. It is always cheaper in the long run to get it right first time.

\section{The patient should be as prepared as possible}

The patient should know what the consultation is to be about. In my outpatient (office) consultations, about half of the new patients do not even know that they have been referred by their general practitioner. None of the others have been given a copy of the referral letter. This means the patient does not know in advance what the consultation is about and cannot even prepare an account of their symptoms or concerns. I believe the patient should have a copy of the referral letter and should be actively encouraged to provide information useful for the consultation. I am sure we could help patients to be better prepared to give an account of their symptoms, concerns, medications, past medical history and tests results. For review-of-progress consultations,
I believe there is far more that we should do to help patients prepare for consultations. For example, there is a wide-ranging set of possible problems that a patient with Parkinson's disease might be experiencing and we could help patients to prepare their information. After each consultation, we should provide the patient with a copy of the clinical letter so that the patient can show this to another clinician. The patient should also know what is likely to happen during the consultation - will they be subject to intimate examination or will the consultation be only a conversation?

\section{The clinician should be as prepared as possible}

The clinical consultation is a serious piece of work; the clinician should be calm, unhurried and mentally prepared for serious work. The clinician should be aware of the whole workload for the session. I prepare for clinic a week in advance by typing an MS Word document that contains known diagnoses, medications and recent results. I can then anticipate the consultation and appear well briefed to the patient. If another patient is slipped into the clinic, I would then spend several minutes reading the notes (charts) before calling the patient in. I do much the same for rounds. I check my patient census on arriving at work and prepare an MS Word document with similar information. I read the current admission notes (or hear the junior doctor present the case) and look up test results etc before going to see the patient. In this way, I can give the patient confidence that at least I know what other clinicians have concluded about their case and I know what to be sceptical about!

\section{The clinician should know the person before making the person into a patient}

I believe this is crucial to correct diagnosis. Knowing the person means asking about the person's job, interests, family and enjoyments. This provides not only an ordinary human connection but makes the person confident that the clinician is interested in them as a person. The patient's account of the presenting complaint is crucial to correct diagnosis. To give their account, the patient must speak. Asking the following easy to answer questions gets the patient talking.

$>$ What is or was your job?

$>$ What are your interests?

$>$ What do you enjoy these days?

$>$ Who is at home with you?

In my experience, the patient then divulges a fuller, richer history of presenting complaint because the patient is confident the clinician is interested and trustworthy. Also, this gives me a memory hook to recall the person and help to be able to recall a whole lot about the diagnosis and treatment. It also often provides interesting conversations at the end of the consultation when I am writing out request forms etc.

\section{The consultation should feel unhurried for the patient and clinician}

If the patient feels hurried by the clinician, they are unlikely to provide a useful history of presenting complaint and will feel that they have not been listened to. When I ask patients to tell medical students 'What makes for a good doctor?' by far and 
away the commonest answer is 'A doctor who listens.' A seemingly hurried clinician cannot appear to be listening. If the clinician feels hurried, they are distracted at a time when attentive listening and clinical reasoning are essential. In the UK, the emphasis on targets in the ED, wards and outpatients (office) means that clinicians feel hurried the majority of the time. In the long run, it is counter-productive to hurry the first consultation. The patient must have confidence that their assessment has been thorough. I am sure that I can discharge more patients from clinic after one attendance because I try to make the consultation unhurried.

\section{The clinician should be able to give undivided} attention to the patient

Attentive listening and clinical reasoning require simultaneously doing two high-level mental activities. Even having to write notes takes up more of the brain's attention. This is one reason that I prepare the MS Word documents prior to the consultation, so that I have less writing or typing to do during the consultation. As I get older and more experienced, I find myself writing less and less during the consultation, so my brain is free to listen, observe and think more. This means that I must not be interrupted or I will lose my chain of thought and forget important points when I compose the clinic letter. I also try to make myself put my mobile phone onto silent, switch off the email on the computer and make the room (or ward) as quiet as possible. It must be exceptionally difficult for consultants in the ED to work on diagnosis; I see them constantly interrupted during consultations, and even dealing with interruptions upon interruptions upon interruptions all in the midst of noise. Ward rounds are also subject to constant noise, interruptions and distractions. We expect correct diagnoses and reviews, but provide almost perfect conditions for human error.

\section{The clinician should be able to hear themself think}

This is a combination of external and internal factors. Externally, everything should be done to provide an environment conducive to high-level thinking. Most clinicians will be laughing by now. On the wards, vacuum cleaners are running, phones are ringing, pagers are bleeping, monitoring equipment is alarming, trolleys trundle and rattle by, staff and relatives are talking at the top of their voices to be heard over the background noise, bed managers, infection control nurses etc all charge into the wards and interrupt.

The attention required to handle the computer programs or locate the current notes and nursing charts to find important information can further detract from being able to hear oneself think.

Internally, the clinician has to find ways to try to block out the external factors and any internal or personal issues so that their mind is able to hear itself think. Top sports performers are trained in these sorts of techniques to block out the spectators, but I have not heard of similar training for clinicians.

There should be a ready supply of information into the consultation

I believe that better clinicians seek information prior to and during the consultation. I have described how I prepare for the consultation; this fits with the often-given advice of 'Read the notes'. Mostly, the information in the notes is truth but must not be accepted without questioning, at the least the information in the notes tells me what to be sceptical about! During the consultation we need access to information because we cannot hold it all in our brains: what was the pro-brain natriuretic peptide level, the creatinine and potassium level, what did electrocardiography and echocardiography show, should the dose of apixaban be reduced in renal impairment? This information can be readily available in wellfiled paper notes, but with the under-provision of ward clerks, clinic clerks and secretaries, well organised paper notes are a thing of the past. I have not experienced well-organised electronic charts. In my previous hospital, I had to have 10 software applications open for an outpatient (office) consultation and the paper notes too! This meant that there was not a ready supply of information into the consultation. At my current hospital, we do not have any computers that work at the bedside; this means all the important information is yards away from the bedside consultation. It is even easy to overlook checking the chest X-ray. The NHS is meant to be National but in Scotland, I cannot access any clinical information about a patient from England, Wales or Northern Ireland and the danger in patient care is palpable.

\section{Confidentiality and dignity must be maintained}

On NHS wards, there is close to no confidentiality and dignity during consultations on ward rounds. The curtains are usually pulled around, but everyone in the bay can hear the consultation. Dignity is often fairly minimal with patients expected to accept physical examination in front of several strangers. In Sweden, Valdemar Erling and colleagues, have set up ward round rooms which provide greater privacy. ${ }^{2}$ The consultations that I observed were more like adult conversations between equals than the conversations that happen when the consultant stands towering over the patient in their bed.

Outpatient consultations are usually far better for confidentiality and dignity. However during my career, I have been expected to do a clinic along with about six other doctors and patients all in a room like a railway station waiting room (this was in one of the top hospitals in London). I have also seen clinics where patients were put into gowns and placed on examination couches before the clinician even said 'Hello'.

\section{The clinician should be regularly refreshed}

Refreshments are called refreshments because they refresh the mind, body and spirit. Most clinicians are workaholics and are then subject to performance targets. I know many clinicians who never take lunch and, at the extremes, may work 14 hours without even going to the toilet. The evidence is that a proper refreshment break is needed every 2 hours if we are to maintain performance in high-level mental activity. If healthcare providers want good patient outcomes and patient experience, they have to ensure and insist that staff take full refreshment breaks - not 10 minutes carrying a coffee, answering emails, making referral calls and chasing tests results - a proper gossipy break doing nothing else but eat, drink and chat.

\section{The patient should be encouraged to have an} important other person participate in the consultation

Another person can give the patient reassurance and may also contribute useful information toward diagnosis or management. The other person is also a second set of ears. Another person is almost essential if the patient is suffering from dementia or 
delirium. However, another important person is often discouraged from participating in consultations on ward rounds.

I am sure there are many other quality indicators that could be applied. For example, I am sure that the patient should, by default, be given a copy of the clinic letter or discharge summary along with copies of important results. In 2019, the patient should have online access to, at least, referral letters, clinic letters, discharge summaries and test results.

\section{Summary}

If healthcare providers realised that clinical consultations are the crucial productive process of their business and worked to improve the process of clinical consultation, we could see fewer misdiagnoses with substantial improvements in patient outcomes and experiences, along with happier more fulfilled staff within 2 years from now.

\section{References}

1 Launer J. Is there a crisis in clinical consultations? Postgrad Med J 2017:93:58.

2 Team-based person-centered ward round, 2019. www.wardround.net

Address for correspondence: Dr Gordon Caldwell, Lorn and Islands District General Hospital, Glengallan Road, Oban, Argyll and Bute PA34 4HH, UK.

Email: gordon.caldwell2@nhs.net

\section{Acute care toolkit 15: Managing acute care problems in pregnancy}

Pregnant women can present to any acute hospital service at any time during their pregnancy or the postpartum period, up to 12 months post-delivery. Women may present with acute medical problems that need to be managed differently because of pregnancy, or may present with obstetric syndromes.

This toolkit provides practical guidance on managing women with acute medical problems in pregnancy. It also advises healthcare staff to take the opportunity to ask about a woman's mental wellbeing when she comes to hospital for other reasons during or after pregnancy; women with a history of mental health problems are more likely to develop new symptoms during pregnancy and postpartum.

Essential reading for front-line NHS staff who may be unfamiliar with the normal physiology of pregnancy and/or diseases that present in pregnancy.

\section{Download now at: www.rcplondon.ac.uk/act15}
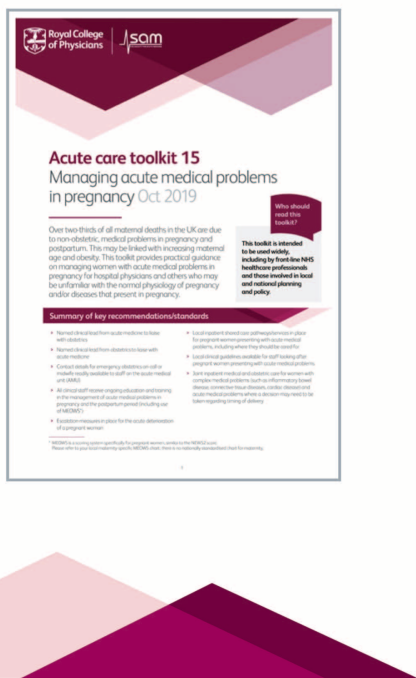